\title{
Alveolar macrophage proteinase/ antiproteinase expression in lung function and emphysema
}

\author{
Takeo Ishii1,2, Raja T. Abboud ${ }^{3}$, Alison M. Wallace ${ }^{1,4}$, John C. English $^{5}$, \\ Harvey 0. Coxson ${ }^{1,6}$, Richard J. Finley ${ }^{4}$, Karey Shumansky ${ }^{1}$, Peter D. Paré ${ }^{1,7}$ \\ and Andrew J. Sandford ${ }^{1,7}$
}

\begin{abstract}
Affiliations: 'UBC James Hogg Research Centre, Institute for Heart and Lung Health, St. Paul's Hospital, Vancouver, BC, ${ }^{3}$ Division of Respiratory Medicine, Vancouver General Hospital, University of British Columbia, Vancouver, BC, ${ }^{4}$ Dept of Surgery, Vancouver General Hospital, University of British Columbia, Vancouver, BC, ${ }^{5}$ Dept of Pathology, Vancouver General Hospital, University of British Columbia, Vancouver, BC, ${ }^{6}$ Dept of Radiology, Vancouver General Hospital, University of British Columbia, Vancouver, BC, and ${ }^{7}$ Dept of Medicine, Division of Respiratory Medicine, University of British Columbia, Vancouver, BC, Canada. ${ }^{2}$ Dept of Internal Medicine, Division of Pulmonary Medicine, Infectious Diseases and Oncology, Nippon Medical School, Tokyo, Japan.
\end{abstract}

Correspondence: A.J. Sandford, UBC James Hogg Research Centre, St. Paul's Hospital, 1081 Burrard Street, Room 166, Vancouver, BC, V6Z 1Y6, Canada. E-mail: Andrew.sandfordahli.ubc.ca

ABSTRACT Alveolar macrophages play an important role in chronic obstructive pulmonary disease via production of matrix metalloproteinases (MMPs) and cathepsins as well as their inhibitors, tissue inhibitors of metalloproteinases and cystatin C. We hypothesised that expression levels of these molecules by alveolar macrophages at baseline and after stimulation would be influenced by genotype and associated with chronic obstructive pulmonary disease phenotypes.

Quantitative PCR and ELISAs/gelatine zymography were used to investigate expression levels of mRNA and protein, respectively. The relationships of expression with genotype, pulmonary function and emphysema were analysed.

The results showed that basal expression level of MMP12 mRNA was inversely related to the diffusing capacity of the lung for carbon monoxide/alveolar volume and to forced expiratory volume in $1 \mathrm{~s} /$ forced vital capacity after correction for multiple comparisons. The expression level of MMP12 protein stimulated with lipopolysaccharide was also inversely related to the diffusing capacity of the lung for carbon monoxide/ alveolar volume and was positively related to the extent of emphysema. The basal expression of MMP1 mRNA was positively correlated with the extent of emphysema. Cathepsin L protein level was positively associated with forced expiratory volume in $1 \mathrm{~s} \%$ predicted.

We conclude that increased MMP12 and MMP1 expression may play a role in the pathogenesis of emphysema. Cathepsin L and MMP9 may be involved in the development of airflow limitation.

@ERSpublications

MMP12 expression may play a role in the pathogenesis of emphysema and airflow limitation http://ow.ly/pICmy

\section{This article has supplementary material available from www.erj.ersjournals.com}

Received: Oct 302012 | Accepted after revision: April 302013 | First published online: July 302013

Support statement: This study was supported by a grant from the Canadian Institutes of Health Research. A.J. Sandford is the recipient of a Michael Smith Foundation for Health Research Senior Scholarship award (grant number CI-SSH01874(07-1)). T. Ishii was the recipient of a Pfizer Fellowship from the Japanese Respiratory Society, and fellowship support from the BC Lung Association, and the CIHR/HSFC IMPACT Strategic Training Program.

Conflict of interest: None declared.

Copyright @ERS 2014 


\section{Introduction}

Chronic obstructive pulmonary disease (COPD) is characterised by airflow limitation that is not fully reversible and is a major cause of chronic morbidity and mortality throughout the world [1]. The hallmark of this disease is an inflammatory process within the lungs in response to noxious particles originating mainly from cigarette smoke, with concomitant destruction and repair of the lung architecture, leading to tissue remodelling. Although the major trigger for the development of COPD is cigarette smoke, only a minority of chronic heavy smokers develop symptomatic COPD [2]. The clustering of COPD in families suggests that there is a genetic component [3] and may explain why only a subset of smokers develops this disease.

A proteinase:antiproteinase imbalance is thought to play a central role in the pathogenesis of COPD [4]. An important source of both proteinases and antiproteinases within the lung is the alveolar macrophage, which produces matrix metalloproteinases (MMPs) and their inhibitors, tissue inhibitors of metalloproteinases (TIMPs), as well as cathepsins and a potent inhibitor of cathepsins, cystatin C (CST3).

MMPs are a family of proteolytic enzymes that degrade collagen or elastin and play an essential role in tissue remodelling and repair associated with inflammation [5]. MMP1, MMP9 and MMP12 are produced by alveolar macrophages, and several studies in animals and humans have provided evidence that these MMPs are important in airway inflammation and the development of emphysema [6, 7]. Cathepsin L (CTSL) and cathepsin S (CTSS) are lysosomal cysteine proteinases produced by alveolar macrophages [6] that may also be involved in COPD through degradation of elastin [8].

As chronic inflammatory processes are thought to be important in the pathology of COPD, cytokines such as interleukin (IL)-1 $\beta$ and tumour necrosis factor (TNF) $-\alpha$ may play a critical role in the disease process [9]. Microbial infections induce exacerbations through bronchial inflammation in COPD patients, which causes tissue damage and could contribute to the development of emphysema [10]. Thus, it is important to also examine the expression change of proteinases and antiproteinases induced by inflammatory cytokines or lipopolysaccharide (LPS), a potent secretagogue for a variety of inflammatory cytokines [10].

We hypothesised that higher expression levels (basal and stimulated by IL-1 $\beta$, TNF- $\alpha$ or LPS) of proteinases (MMP1, MMP9, MMP12, CTSL and CTSS) and/or lower levels of antiproteinases (TIMP1, TIMP2, TIMP3 and CST3) in human alveolar macrophages would be associated with lower lung function (airflow limitation and diffusing capacity of the lung for carbon monoxide (DLCO)) and greater extent of emphysema. We also hypothesised that polymorphisms of these genes would alter the level of expression of their mRNA and protein.

\section{Materials and methods}

Study subjects

109 subjects who underwent lung resection for small ( $<3 \mathrm{~cm}$ diameter), TNM (tumour, node, metastasis) stage I or II, peripheral tumours at Vancouver General Hospital (Vancouver, BC, Canada) were recruited for this study. Partial results from 54 of these subjects were included in a previous study [11]. Preoperative pulmonary function tests were performed including forced expiratory volume in $1 \mathrm{~s}$ (FEV1), forced vital capacity (FVC) and single breath DLCO/alveolar volume (VA), in accordance with American Thoracic Society guidelines [12]. Computed tomography (CT) scans were available for assessment of emphysema in 75 subjects. The characteristics of the subjects are shown in table 1.

This study was approved by the University of British Columbia/Providence Health Care and Vancouver Hospital and Health Sciences Research Ethics Boards (Vancouver, BC, Canada). All subjects provided written informed consent for the study.

\section{Computed tomographic analysis}

Details of a semi-quantitative emphysema visual score, and a quantitative computerised analysis of the percentage of lung with emphysema, are given in the online supplementary materials and methods.

\section{Bronchoalveolar lavage and cell culture}

The resected lung or lobe was obtained immediately post-operatively and bronchoalveolar lavage was performed. Details of the lavage, macrophage separation and subsequent culture are given in the online supplementary materials and methods.

RNA extraction, cDNA synthesis and quantitative real-time PCR

Details of cDNA preparation and quantification by real-time PCR (online supplementary table S1) are given in the online supplementary materials and methods. 


\section{TABLE 1 Characteristics of the study subjects ${ }^{\#}$}

\begin{tabular}{|c|c|}
\hline Age years & $67 \pm 10$ \\
\hline Males & $49(45)$ \\
\hline \multicolumn{2}{|l|}{ Race } \\
\hline Caucasian & $87(80)$ \\
\hline Asian & $17(16)$ \\
\hline Unknown & 5 \\
\hline \multicolumn{2}{|l|}{ Smoking history } \\
\hline Current smokers & $17(16)$ \\
\hline Former smokers & $92(84)$ \\
\hline Pack-years & $46 \pm 25$ \\
\hline \multicolumn{2}{|l|}{ Spirometry } \\
\hline FEV1 \% pred pre-bronchodilator $(n=109)$ & $83 \pm 20$ \\
\hline FEV1 \% pred post-bronchodilator $(n=97)$ & $87 \pm 19$ \\
\hline FVC $\%$ pred pre-bronchodilator $(n=109)$ & $92 \pm 17$ \\
\hline FVC $\%$ pred post-bronchodilator $(n=97)$ & $95 \pm 16$ \\
\hline FEV1/FVC \% pre-bronchodilator $(n=109)$ & $70 \pm 11$ \\
\hline FEV1/FVC $\%$ post-bronchodilator $(n=100)$ & $70 \pm 13$ \\
\hline$D\llcorner c o / V A \%$ pred $(n=81)$ & $75 \pm 20$ \\
\hline $\mathrm{RV} / \mathrm{TLC} \%(\mathrm{n}=73)$ & $44 \pm 10$ \\
\hline \multicolumn{2}{|l|}{ GOLD classification } \\
\hline 0 & 64 \\
\hline 1 & 19 \\
\hline 2 & 20 \\
\hline 3 & 5 \\
\hline 4 & 1 \\
\hline \multicolumn{2}{|l|}{$\alpha_{1}$-antitrypsin genotype ${ }^{\pi}$} \\
\hline MM & 104 (97) \\
\hline MZ & $3(3)$ \\
\hline ZZ & $0(0)$ \\
\hline
\end{tabular}

Data are presented as mean \pm SD, $\mathrm{n}(\%)$ or $\mathrm{n}$. FEV1: forced expiratory volume in $1 \mathrm{~s} ; \%$ pred: \% predicted; FVC: forced vital capacity; DLCO: diffusing capacity of the lung for carbon monoxide; VA: alveolar volume; RV: residual volume; TLC: total lung capacity; GOLD: Global Initiative for Chronic Obstructive Lung Disease. $\#: \mathrm{n}=109 ;{ }^{\top}$ : two subjects were not genotyped because genomic DNA could not be obtained.

\section{Protein quantification}

Details of the protein quantification by ELISAs or zymography are given in the online supplementary materials and methods.

\section{Genotyping}

We selected polymorphisms (online supplementary table S2) in MMPs, cathepsins, TIMPs and CST3 that have been previously associated with COPD and/or with regulation of gene expression ( $r s 1799750$ in MMP1, D20S838 and rs3918242 in MMP9, rs2276109 in MMP12, and rs2277698 in TIMP2 [13-17]. In addition, we selected single nucleotide polymorphisms (SNPs) from the HapMap Project (www.hapmap. org) to identify a haplotype block covering the promoter region of each gene (details are given in the online supplementary materials and methods).

\section{Statistical analysis}

Statistical analyses were performed using JMP software version 5.1 (SAS Institute Inc., Cary, NC, USA). Linear regression analysis was used to evaluate mRNA or protein expression versus pulmonary function tests and quantitative CT scan data. We also used linear regression to evaluate genotype versus mRNA or protein expression with an additive model, and p-values were calculated in the linear model based on a Wald test statistic. Ordinal regression was used to examine the association between semiquantitative CT scores and gene expression levels. Adjustment was performed for age, sex, race and smoking history (pack-years). If the values were not normally distributed, they were log-transformed before analyses.

p-values $<0.05$ were considered nominally significant. Due to the nature of the analysis and the multipletesting of independent and dependent variables, modified Bonferroni correction for multiple comparisons was done as described by NYHOLT [18] and details of this correction procedure are provided in the online 
supplementary tables S3 and S4. The power of this study design was estimated using the many proportions function and linear regression function in PASS 2005 [19].

\section{Results}

Proteinase and antiproteinase mRNA expression

The mRNA expression levels of the proteinases (MMP1, MMP9, MMP12, CTSL and CTSS) and antiproteinases (TIMP1, TIMP2, TIMP3 and CST3) in alveolar macrophages under the different experimental conditions (basal, i.e. uncultured macrophages, cultured without stimulation, cultured with stimulation by IL$1 \beta$, TNF- $\alpha$ or LPS) are shown in online supplementary figures S1-S9. The alveolar macrophages cultured without stimulation showed a significant increase in expression of all of the genes (except TIMP3 and CST3) relative to the basal gene expression level. With LPS stimulation, the expression levels of all the proteases (except CTSS), as well as TIMP1, were significantly increased. Conversely, the expression levels of TIMP 2 and CST3 were significantly decreased after LPS stimulation. Following stimulation with either IL-1 $\beta$ or TNF- $\alpha$ the expression levels of MMP1, MMP9 and TIMP1 were significantly increased. Stimulation with either IL-1 $\beta$ or TNF- $\alpha$ resulted in significantly decreased TIMP2 expression.

There were no significant effects of age, sex or race on the basal expression of the genes. The effect of smoking status on basal gene expression is shown in online supplementary table S5. MMP12 basal expression was higher and TIMP2 basal expression was lower in current smokers compared with former smokers. TIMP3 basal expression was positively correlated with pack-years while CTSS expression was inversely correlated with pack-years.

Proteinase and antiproteinase genotypes versus basal and stimulated gene expression As genetic variation could affect COPD phenotypes through modulation of the proteinase and antiproteinase mRNA and protein expression profiles, we investigated whether polymorphisms altered expression levels of their respective gene products. All of the polymorphisms we investigated were in Hardy-Weinberg equilibrium $(p \geqslant 0.18)$ in the Caucasian subgroup $(n=87)$, as shown in online supplementary table S2.

A summary of the results of association between genotypes and basal gene expression and expression change is shown in online supplementary table S6. MMP1 mRNA basal expression and protein expression induced by LPS were both inversely associated with increasing number of the $2 \mathrm{G}$ allele of MMP1 rs1799750 (online supplementary figs S10 and S11, respectively). CTSL protein expression without stimulation was inversely associated with increasing number of the T allele of CTSL rs2274611 (online supplementary fig. S12). The most significant association of genotype with gene expression was observed for the basal expression of CST3 mRNA, which was inversely associated with increasing number of the A allele of CST3 rs6036478 (online supplementary fig. S13). All of these associations remained significant after correction for multiple comparisons.

\section{Proteinase and antiproteinase mRNA expression and COPD phenotypes}

We analysed the relationship of COPD phenotypes and basal mRNA expression levels using linear regression analyses (table 2 and online supplementary table S7). The basal expression level of MMP12 in alveolar macrophages showed a significant inverse association with $D \mathrm{LCO} / \mathrm{VA} \%$ predicted that remained significant after correction for multiple comparisons. A scatter plot of the association between MMP12 basal mRNA expression and DLCO/VA \% pred is shown in figure 1 . There was also an inverse association between basal MMP12 and FEV1/FVC \% (fig. 2). The data also suggest that there was a positive association $(\mathrm{p}=0.0052$, uncorrected for multiple comparisons) between basal expression of $M M P 1$ and the semiquantitative visual assessment of extent of emphysema of all CT scans (total $n=75$ ); this association was no longer significant after correction for multiple comparisons, as the level of significance required was $\mathrm{p}<0.0012$. There was no significant association (uncorrected $\mathrm{p}=0.120$ ) with the computerised quantitative assessment of emphysema by CT scanning $(n=45)$, which may be due to the lower number of subjects with these data available.

We also tested for associations between changes in mRNA expression induced by LPS, TNF- $\alpha$ and IL-1 $\beta$ and COPD phenotypes (table 3 and online supplementary table S8). The most significant result was that expression change of MMP9 mRNA stimulated by TNF- $\alpha$ was inversely associated with FEV1 \% pred $(\mathrm{p}=0.0005)$, but this did not survive correction for multiple comparisons, since the level of significance required was $\mathrm{p}<0.00042$.

\section{The relationship between mRNA and protein expression}

As we postulated that gene expression would be associated with COPD phenotypes through the level of protein expression, we investigated the association between mRNA and protein expression for the genes that 
TABLE 2 Significant results $(p<0.05)$ from linear regression analysis of pulmonary function tests and computed tomography-scan data with mRNA expression at baseline

\begin{tabular}{lccc} 
Gene & Outcome & $\mathbf{r}^{\mathbf{2}}$ & p-value \\
\hline MMP1 & Semiquantitative score for emphysema & 0.0597 & 0.0052 \\
MMP12 & FEV1 \% pred & 0.2565 & 0.0265 \\
& FEV1/FVC \% & 0.2393 & $\mathbf{8} \times \mathbf{1 0 ^ { - 5 }}$ \\
TIMP2 & DLCO/VA \% pred & 0.2112 & $\mathbf{0 . 0 0 0 4}$ \\
CST3 & FEV1\% pred & 0.2547 & 0.0299 \\
& FEV1 \% pred & 0.2736 & 0.0087 \\
\hline
\end{tabular}

MMP: metalloproteinase; TIMP: tissue inhibitors of metalloproteinase; CST3: cystatin C; FEV1: forced expiratory volume in $1 \mathrm{~s}$; \% pred: \% predicted; FVC: forced vital capacity; DLCO: diffusing capacity of the lung for carbon monoxide; $V_{A}$ : alveolar volume. Coefficients of determination $\left(r^{2}\right)$ and $p$-values were calculated from regression analysis of each clinical outcome including age, sex, race, pack-years and mRNA expression as covariates. $r^{2}$ values are for the whole model, $p$-values are for the mRNA expression level. FEV $1 \%$ pred and FEV1/FVC \% were measured post-bronchodilator. An ordinal logistic regression was performed for the semiquantitative emphysema scores. DLCo/VA \% pred was log-transformed. Significant results after correction for multiple comparisons are shown in bold. The $p$-value threshold is $0.05 /(9 \times 4.45)=0.0012$. Complete results are shown in online supplementary table S7.

showed at least nominally significant associations with the clinical outcomes. We compared the mRNA levels at $24 \mathrm{~h}$ with the protein concentrations of MMP1, MMP9, MMP12, CTSL, TIMP2 and CST3 in media after $48 \mathrm{~h}$ of culture. The levels of protein are shown in online supplementary table S9. We observed significant correlations between the mRNA and protein levels for MMP1 stimulated with LPS, MMP9 with and without LPS, and MMP12 with and without LPS (online supplementary table S10). The correlations between MMP12 mRNA and protein expression of MMP12 in alveolar macrophages are shown in figure 3. The basal mRNA expression of MMP12 was also positively correlated with its protein expression level stimulated with LPS $\left(\mathrm{p}=1 \times 10^{-5}\right)$.

We also investigated the correlation between the fold change in mRNA and protein levels after culture with the various stimuli (online supplementary table S11). Significant relationships were observed for changes in MMP1 level after stimulation with LPS and IL- $\beta$. The changes in expression of MMP9 mRNA and protein after culture with all the stimuli were also significantly correlated.

\section{Protein expression of proteinases and antiproteinases and COPD phenotypes}

The protein expression of the proteinases and antiproteinases was investigated in relation to COPD phenotypes (table 4 and online supplementary table S12). The protein expression of MMP12 stimulated with LPS was inversely associated with $D \mathrm{LCO} / V_{\mathrm{A}} \%$ predicted and positively associated with quantitative score for emphysema. The protein expression of CTSL with or without LPS was also positively associated with FEV1 \% predicted. However, none of these associations with protein expression were significant after correction for multiple comparisons.

\section{Discussion}

In this study, we found that the genotypes of MMP1, CTSL and CST3 showed significant associations with their expression at the mRNA and/or protein level. The MMP1 1G/2G polymorphism (rs1799750) has previously been associated with gene expression [20], but this is the first description of an effect on transcription in alveolar macrophages. There are no previous studies of the rs2274611 CTSL polymorphism, but a CTSL promoter SNP (rs3118869) has been associated with reporter gene expression in vitro [21]. The C allele of $\mathrm{rs} 3118869$ showed greater expression than the A allele $\left(\mathrm{p}=3.36 \times 10^{-6}\right)$ in luciferase assays and was associated $(p=0.0143)$ with a higher level of CTSL protein in our study (online supplementary table S6). The $\mathrm{C}$ allele of $\mathrm{rs} 3118869$ is in high linkage disequilibrium $\left(\mathrm{r}^{2}=0.8648\right)$ with the $\mathrm{C}$ allele of rs2274611, which was associated $(p=0.0007)$ with higher CTSL protein in our study (online supplementary table S6). Thus, this is the first study to confirm the functional effect of rs3118869 on transcription and we also demonstrated a novel association with CTSL protein level. There are no previous studies of the CST3 rs6036478 polymorphism in the literature, but this SNP is part of a haplotype of SNPs in high linkage disequilibrium (all $\mathrm{r}^{2}>0.92$ ), which has been shown to influence serum/plasma CST3 levels [22-25]. Our observation that the A (minor) allele of rs6036478 was associated with lower basal CST3 mRNA (online supplementary table S6) is consistent with these previous data and is novel in the context of COPD. 
FIGURE 1 The relationship between basal mRNA expression of matrix metalloproteinase 12 (MMP12) in alveolar macrophages and diffusing capacity of the lung for carbon monoxide (DLCO)/alveolar volume (VA) $\%$ predicted (\% pred).

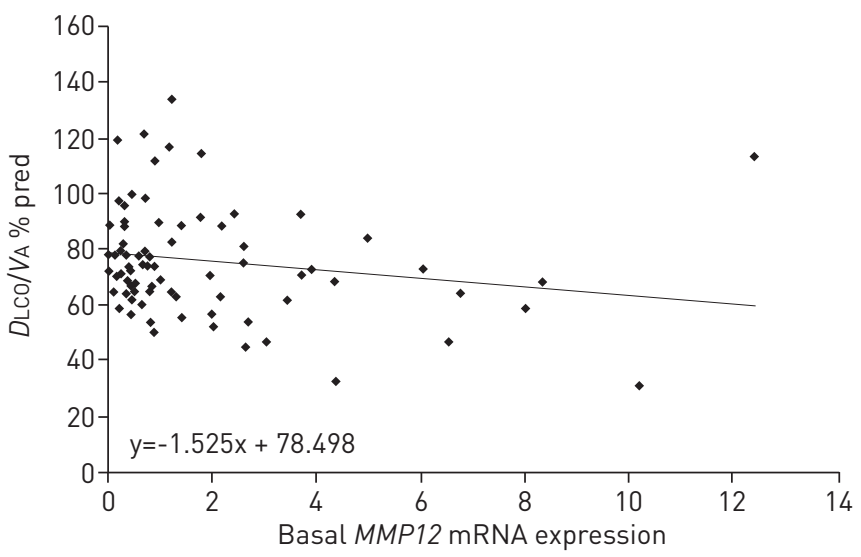

The basal level of MMP12 mRNA expression was inversely related to DLCO/VA \% pred and FEV1/FVC \% (table 2). MMP12 protein level after stimulation with LPS was also inversely related to DLCO/VA \% pred and was positively associated with the quantitative score for emphysema (table 4 and online supplementary table S12). Although these latter associations were not significant after correction for multiple comparisons, the concordance of direction of association suggests a true pathophysiological role for this proteinase.

Our novel findings of associations between MMP12 expression and lung diffusing capacity, as well as the extent of emphysema, suggest that MMP12 has a role in the development of emphysema in humans. It has been reported that genetic variation in MMP12 is associated with COPD and asthma [26]. However, there are no reports on the direct association between emphysema and the expression of MMP12 from alveolar macrophages in humans. MMP12 knockout mice do not develop emphysema following exposure to cigarette smoke [27], suggesting that the presence of MMP12 is critical in smoke-induced emphysema in mice. Our study further suggests a role for MMP12 in the pathogenesis of emphysema in humans. The underlying mechanism for this role may relate to MMP12 stimulation of neutrophils through activation of TNF- $\alpha$, resulting in the production of MMP9, neutrophil elastase and cathepsin G, all of which degrade elastin and may lead to emphysema [28]. In addition, MMP9 and MMP12 degrade specific collagens $[29,30]$. MMP12 is increased in the lungs [31] and sputum [32] of COPD patients.

We also showed that the basal level of $M M P 1$ mRNA was positively correlated with a semiquantitative assessment of the extent of emphysema $(\mathrm{p}=0.0052)$, as in our previous report, based on the first 44 cases with CT scans [11]. Thus, we have extended our previous observations by demonstrating that not only MMP12 mRNA but also protein level is associated with DLCO/VA and emphysema.

Cigarette smoke and other irritants stimulate alveolar macrophages to produce MMPs [33, 34] and cathepsins [35]. MMP1 degrades collagen whereas MMP9, MMP12, CTSL and CTSS degrade elastin [36]. Although human MMP1 is a collagenase, its expression in the lungs of transgenic mice caused emphysema [37] and expression of MMP1 was increased in the lungs of patients with emphysema [38]. Smokers who have airway obstruction show increased expression of MMP1 and MMP9 compared with smokers without COPD and nonsmokers [39].

FIGURE 2 The relationship between basal mRNA expression of matrix metalloproteinase 12 (MMP12) in alveolar macrophages and forced expiratory volume in $1 \mathrm{~s}$ (FEV1)/ forced vital capacity (FVC) \%.

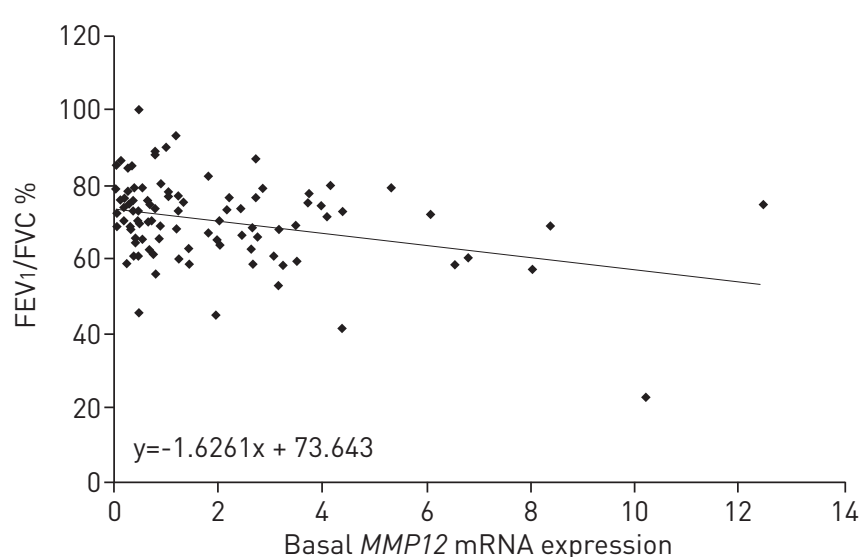


TABLE 3 Significant results $(p<0.05)$ from linear regression analysis of pulmonary function tests and computed tomography-scan data with changes in mRNA in response to stimuli

\begin{tabular}{|c|c|c|c|c|}
\hline Gene & Stimulus & Outcome & $r^{2}$ & p-value \\
\hline MMP9 & TNF- $\alpha$ & FEV1 \% pred & 0.3150 & 0.0005 \\
\hline CTSL & LPS & Semiquantitative score for emphysema & 0.0653 & 0.0162 \\
\hline TIMP1 & LPS & FEV $1 / F V C \%$ & 0.1445 & 0.0219 \\
\hline TIMP3 & LPS & Semiquantitative score for emphysema & 0.0582 & 0.0407 \\
\hline
\end{tabular}

MMP: metalloproteinase; CTSL: cathepsin L; TIMP: tissue inhibitor of metalloproteinase; TNF- $\alpha$ : tumour necrosis factor- $\alpha$; LPS: lipopolysaccharide; FEV1: forced expiratory volume in $1 \mathrm{~s} ; \%$ pred: \% predicted; FVC: forced vital capacity. The mRNA was obtained after $24 \mathrm{~h}$ of culture with the various stimuli, and the change of mRNA from cells cultured with no stimuli was analysed. Coefficients of determination $\left(r^{2}\right)$ and $p$-values were calculated from regression analysis of each clinical outcome including age, sex, race, pack-years and mRNA expression as covariates. $r^{2}$ values are for the whole model, $p$-values are for the mRNA expression level. FEV1 $\%$ pred and FEV1/FVC \% were measured post-bronchodilator. An ordinal logistic regression was performed for the semiquantitative emphysema scores. The $p$-value threshold to correct for multiple corrections is $0.05 /[9$ (number of single nucleotide polymorphisms) $\times 3$ (number of conditions) $\times 4.45$ (number of phenotypes) $)=0.00042$. Complete results are shown in online supplementary table $\mathrm{S} 8$.

We observed a novel association of TNF- $\alpha$ induced MMP9 mRNA expression with FEV1\% pred (table 3). This observation is consistent with previous data that demonstrated MMP9 expression increased in the lung parenchyma surrounding small airways as FEV1 \% pred decreased [40]. There is also supportive evidence that MMP9 contributes to the development of emphysema from mouse models [41, 42]. Our data suggest that it is the level of MMP9 in response to specific pro-inflammatory stimuli that plays a critical role in the development of COPD, in agreement with studies showing that increases in TNF- $\alpha$ are associated with reductions in lung function [40] and that TNF- $\alpha$ plays an important role in cigarette smoke-induced emphysema [43].

CTSL protein level was associated with $\mathrm{FEV}_{1} \%$ pred, which suggests that this proteinase has a role in development of COPD. However, individuals whose macrophages secreted more CTSL had better lung function. We did not observe this relationship in our previous study [11] but the same trends were present (i.e. higher CTSL with or without LPS associated with higher lung function) with similar estimates from the regression analysis. However, the p-values were not significant due to the smaller sample size. It is unclear what mechanism underlies this "paradoxical" relationship. However, CTSL expression in thymocytes is essential for natural killer T-cell development [44], and natural killer cells, which have an essential role in immunity against viral infection [45], are decreased in the peripheral blood in COPD [46]. Similarly, CTSL is involved in antigen processing $[47,48]$ and T-helper cell development $[49,50]$. Thus, CTSL protein expression could reinforce immunological capacity, reduce exacerbations and prevent progression of COPD. CSTL also plays a prominent role in the production of neuropeptides [51], such as neuropeptide Y,
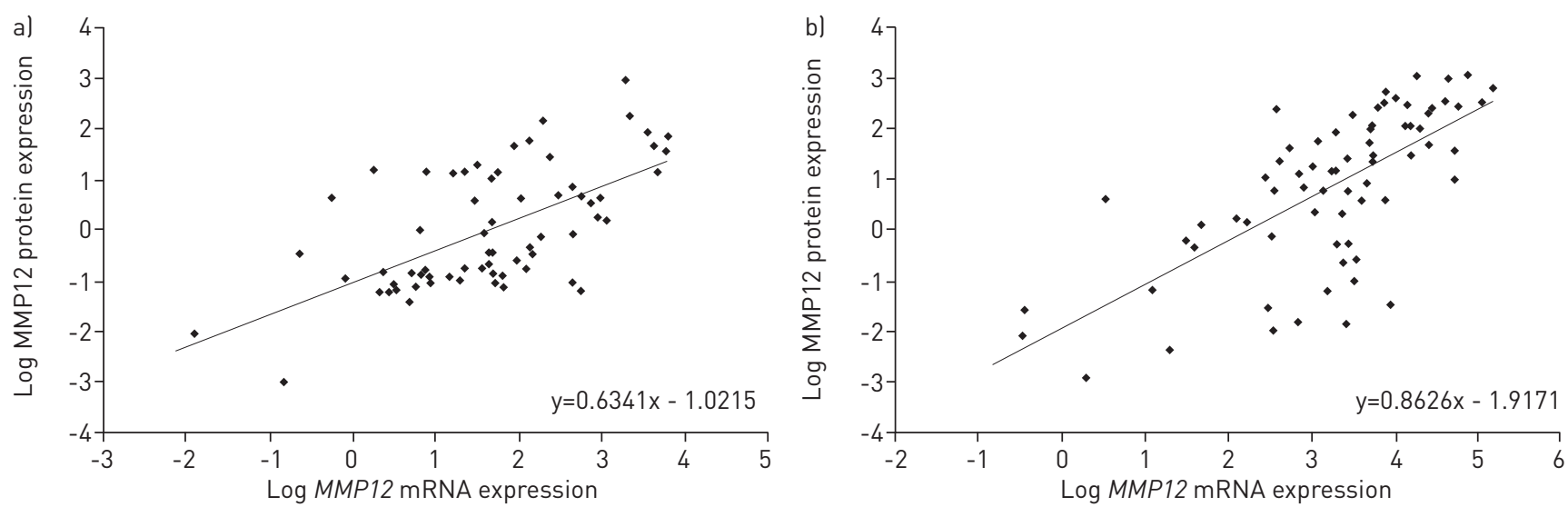

FIGURE 3 The correlation between MMP12 mRNA and protein expression of matrix metalloproteinase (MMP)12 in alveolar macrophages. The mRNA expression level of MMP12 at $24 \mathrm{~h}$ was positively correlated with MMP12 protein in the media in which alveolar macrophages were cultured at 48 h. a) No stimulation and b) stimulation with lipopolysaccharide. 
TABLE 4 Significant results $(p<0.05)$ from linear regression of COPD phenotypes with protein expression of proteinases and antiproteinases

\begin{tabular}{lcccc} 
Gene & Condition & Outcome & $\mathbf{r}^{\mathbf{2}}$ & $\mathbf{p}$-value \\
\hline \multirow{2}{*}{ MMP12 } & with LPS & DLCo/VA \% pred & 0.2173 & 0.0449 \\
MMP12 & with LPS & Quantitative score for emphysema & 0.4457 & 0.0326 \\
CTSL & without LPS & FEV1 \% pred & 0.2163 & 0.0447 \\
CTSL & with LPS & FEV1\% pred & 0.2251 & 0.0262 \\
\hline
\end{tabular}

MMP: metalloproteinase; CTSL: cathepsin L; LPS: lipopolysaccharide; DLCO: diffusing capacity of the lung for carbon monoxide; VA: alveolar volume; \% pred: \% predicted; FEV1: forced expiratory volume in $1 \mathrm{~s}$. The protein was obtained after $48-\mathrm{h}$ culture with various stimuli in the media, and the associations between protein concentration and chronic obstructive pulmonary disease (COPD) phenotypes were analysed by regression. Adjusted by age, sex, race, and pack-years. FEV1 \% pred and FEV1/forced vital capacity \% were measured postbronchodilator. For the semiquantitative emphysema scores an ordinal logistic regression was performed. DLCO/VA \% pred and quantitative score for emphysema deviated from the normal distribution and were logtransformed. The p-value threshold after Nyholt's correction is $0.05 / 16$ (number of single nucleotide polymorphisms) $\times 2$ (number of conditions) $\times 4.45$ (number of phenotypes) $=0.00094$. MMP12 level after stimulation with LPS was inversely related to DLCO/VA \% pred and was positively associated with quantitative score for emphysema. CTSL levels without and with LPS stimulation were both positively related to FEV $1 \%$ pred. Complete results are shown in online supplementary table S8.

which is decreased in the airway epithelium in COPD patients compared with unaffected smokers [52], suggesting a protective role for this molecule. In addition, CSTL is involved in the production of adrenocorticotropic hormone, and upregulation of this hormone is attenuated in COPD patients following exercise [53].

In this study, there were significant differences in gene expression in macrophages cultured without stimulation compared with primary macrophages, as we have previously shown [11]. This phenomenon has also been observed in murine macrophages [54] and other cell types following culture [55, 56]. While it was not surprising that nonphysiological conditions (i.e. cell culture) altered gene expression in our study, whether this was due to adherence of the gcells to the plastic substrate in the cell culture dishes or to components of the culture medium is not known.

A recent study investigated changes in gene expression following instillation of LPS into the lung in healthy individuals $(n=8)$ compared with instillation of saline into the contralateral lung [57]. In that in vivo study [57], TIMP1 was upregulated 2.4-fold $\left(\mathrm{p}=3 \times 10^{-4}\right)$ following challenge with LPS, mirroring the increase in TIMP1 mRNA that we observed after culture with LPS (online supplementary fig. S6). Similarly, TIMP2 mRNA was downregulated following instillation of LPS $\left(\mathrm{p}=3 \times 10^{-3}\right)$, and we also observed a significant decrease in TIMP2 mRNA following culture with LPS (online supplementary fig. S7).

In comparison with our previous study [11], we have doubled the sample size to increase the power of the analyses and have assessed additional genes (i.e. the antiproteinases TIMP1, TIMP2, TIMP3 and CST3). We also measured the levels of the proteins corresponding to six of the genes whereas our previous manuscript only included mRNA data [11].

There are some limitations of our study with respect to gene expression and COPD or emphysema phenotypes. With a total sample of 109 subjects we have $80 \%$ power to detect associations between gene expression and COPD phenotypes only where the $r^{2}$ value is $\geqslant 0.3$. As we have a smaller number of subjects for $\mathrm{DLCO} / \mathrm{VA} \%$ pred and the CT scan data, the power associated with these phenotypes is less. In addition, there was a small proportion of severe COPD patients in our study ( $59 \%$ of our subjects had FEV1 values within the normal range). In addition, the use of alveolar macrophages from patients with lung cancer adds a potential bias to our study. Specifically, several MMP polymorphisms have been associated with lung cancer [58] and, therefore, the risk alleles may have been selected for in our study group. However, all SNPs in our study showed good agreement with expectations under Hardy-Weinberg equilibrium (online supplemetary table S2) suggesting that there was no strong selection bias. In addition, the allele frequencies are generally similar to the HapMap data (online supplemetary table S2). Expression levels of proteinase and antiproteinase genes may also have been affected by the presence of tumours in the lungs. However, as noted above, our results are similar to those of REYNIER et al. [57] in subjects without cancer, suggesting that the observations in this study are applicable to noncancer populations. 
In conclusion, among proteinases and antiproteinases, our data further support previous studies demonstrating that MMP12 and MMP1 may have an important role in the pathogenesis of emphysema. In addition, CTSL may positively influence the development of airflow limitation through protein expression and is affected by genetic variation.

\section{Acknowledgements}

The authors gratefully acknowledge the technical assistance of Hong Li, Rosa Garcia, Beth Whalen, Sebastian Cogswell, Barbara Moore, Mark Elliot and Steve Kalloger (University of British Columbia, Vancouver, BC, Canada). We also gratefully acknowledge Ken Evans and John Yee (University of British Columbia) for providing us with lung specimens from their patients undergoing lung resection surgery, as well as Paola Nasute Fauerbach and Nestor Müller (University of British Columbia) for assigning the semiquantitative emphysema scores to the CT scans.

Peter D. Paré is a Michael Smith Distinguished Scholar and the Jacob Churg Distinguished Researcher.

\section{References}

1 Murray CJ, Lopez AD. Alternative projections of mortality and disability by cause 1990-2020: Global Burden of Disease Study. Lancet 1997; 349: 1498-1504.

2 Antó JM, Vermeire P, Vestbo J, et al. Epidemiology of chronic obstructive pulmonary disease. Eur Respir J 2001; 17: 982-994.

3 McCloskey SC, Patel BD, Hinchliffe SJ, et al. Siblings of patients with severe chronic obstructive pulmonary disease have a significant risk of airflow obstruction. Am J Respir Crit Care Med 2001; 164: 1419-1424.

4 Hogg JC, Senior RM. Chronic obstructive pulmonary disease - part 2: pathology and biochemistry of emphysema. Thorax 2002; 57: 830-834.

Nagase H, Woessner JF. Matrix metalloproteinases. J Biol Chem 1999; 274: 21491-21494.

Shapiro SD. The macrophage in chronic obstructive pulmonary disease. Am J Respir Crit Care Med 1999; 160: S29-S32.

7 Mocchegiani E, Giacconi R, Costarelli L. Metalloproteases/anti-metalloproteases imbalance in chronic obstructive pulmonary disease: genetic factors and treatment implications. Curr Opin Pulm Med 2011; 17: Suppl. 1, S11-S19.

8 Chapman HA, Riese RJ, Shi GP. Emerging roles for cysteine proteases in human biology. Annu Rev Physiol 1997; 59: 63-88.

9 Chung KF. Cytokines in chronic obstructive pulmonary disease. Eur Respir J 2001; 18: Suppl. 34, 50s-59s.

10 Wilson R. Bacteria, antibiotics and COPD. Eur Respir J 2001; 17: 995-1007.

11 Wallace AM, Sandford AJ, English JC, et al. Matrix metalloproteinase expression by human alveolar macrophages in relation to emphysema. COPD 2008; 5: 13-23.

12 American Thoracic Society. Single-breath carbon monoxide diffusing capacity (transfer factor). Recommendations for a standard technique - 1995 update. Am J Respir Crit Care Med 1995; 152: 2185-2198.

13 Joos L, He JQ, Shepherdson MB, et al. The role of matrix metalloproteinase polymorphisms in the rate of decline in lung function. Hum Mol Genet 2002; 11: 569-576.

14 Minematsu N, Nakamura H, Tateno H, et al. Genetic polymorphism in matrix metalloproteinase- 9 and pulmonary emphysema. Biochem Biophys Res Commun 2001; 289: 116-119.

15 Zhou M, Huang SG, Wan HY, et al. Genetic polymorphism in matrix metalloproteinase-9 and the susceptibility to chronic obstructive pulmonary disease in Han population of south China. Chin Med J (Engl) 2004; 117: 1481-1484.

16 Shimajiri S, Arima N, Tanimoto A, et al. Shortened microsatellite d(CA)21 sequence down-regulates promoter activity of matrix metalloproteinase 9 gene. FEBS Lett 1999; 455: 70-74.

17 Hirano K, Sakamoto T, Uchida Y, et al. Tissue inhibitor of metalloproteinases-2 gene polymorphisms in chronic obstructive pulmonary disease. Eur Respir J 2001; 18: 748-752.

18 Nyholt DR. A simple correction for multiple testing for single-nucleotide polymorphisms in linkage disequilibrium with each other. Am J Hum Genet 2004; 74: 765-769.

19 Hintze J. NCSS and PASS. 2004. Number Cruncher Statistical Systems. Kaysville, UT. www.ncss.com. Date last accessed: June 5, 2013.

20 Arakaki PA, Marques MR, Santos MC. MMP-1 polymorphism and its relationship to pathological processes. J Biosci 2009; 34: 313-320.

21 Mbewe-Campbell N, Wei Z, Zhang K, et al. Genes and environment: novel, functional polymorphism in the human cathepsin L (CTSL1) promoter disrupts a xenobiotic response element (XRE) to alter transcription and blood pressure. J Hypertens 2012; 30: 1961-1969.

22 Mitaki S, Nagai A, Sheikh AM, et al. Contribution of cystatin C gene polymorphisms to cerebral white matter lesions. Cerebrovasc Dis 2011; 32: 489-496.

23 Eriksson P, Deguchi H, Samnegård A, et al. Human evidence that the cystatin C gene is implicated in focal progression of coronary artery disease. Arterioscler Thromb Vasc Biol 2004; 24: 551-557.

24 Benussi L, Ghidoni R, Steinhoff T, et al. Alzheimer disease-associated cystatin C variant undergoes impaired secretion. Neurobiol Dis 2003; 13: 15-21.

25 Loew M, Hoffmann MM, Koenig W, et al. Genotype and plasma concentration of cystatin C in patients with coronary heart disease and risk for secondary cardiovascular events. Arterioscler Thromb Vasc Biol 2005; 25: 1470-1474.

26 Hunninghake GM, Cho MH, Tesfaigzi Y, et al. MMP12, lung function, and COPD in high-risk populations. N Engl J Med 2009; 361: 2599-2608.

27 Hautamaki RD, Kobayashi DK, Senior RM, et al. Requirement for macrophage elastase for cigarette smoke-induced emphysema in mice. Science 1997; 277: 2002-2004.

28 Churg A, Wang RD, Tai $\mathrm{H}$, et al. Macrophage metalloelastase mediates acute cigarette smoke-induced inflammation via tumor necrosis factor-alpha release. Am J Respir Crit Care Med 2003; 167: 1083-1089.

29 Okada Y, Gonoji Y, Naka K, et al. Matrix metalloproteinase 9 (92-kDa gelatinase/type IV collagenase) from HT 1080 human fibrosarcoma cells. Purification and activation of the precursor and enzymic properties. J Biol Chem 1992; 267: 21712-21719. 
Gronski TJ Jr, Martin RL, Kobayashi DK, et al. Hydrolysis of a broad spectrum of extracellular matrix proteins by human macrophage elastase. J Biol Chem 1997; 272: 12189-12194.

31 Molet S, Belleguic C, Lena $\mathrm{H}$, et al. Increase in macrophage elastase (MMP-12) in lungs from patients with chronic obstructive pulmonary disease. Inflamm Res 2005; 54: 31-36.

32 Demedts IK, Morel-Montero A, Lebecque S, et al. Elevated MMP-12 protein levels in induced sputum from patients with COPD. Thorax 2006; 61: 196-201.

33 Russell REK, Culpitt SV, DeMatos C, et al. Release and activity of matrix metalloproteinase-9 and tissue inhibitor of metalloproteinase-1 by alveolar macrophages from patients with chronic obstructive pulmonary disease. Am $\mathrm{J}$ Respir Cell Mol Biol 2002; 26: 602-609.

34 Bracke K, Cataldo D, Maes T, et al. Matrix metalloproteinase-12 and cathepsin D expression in pulmonary macrophages and dendritic cells of cigarette smoke-exposed mice. Int Arch Allergy Immunol 2005; 138: 169-179.

35 Reilly JJ Jr, Chen P, Sailor LZ, et al. Cigarette smoking induces an elastolytic cysteine proteinase in macrophages distinct from cathepsin L. Am J Physiol 1991; 261: L41-L48.

36 Parks WC, Shapiro SD. Matrix metalloproteinases in lung biology. Respir Res 2001; 2: 10-19.

37 D'Armiento J, Dalal SS, Okada Y, et al. Collagenase expression in the lungs of transgenic mice causes pulmonary emphysema. Cell 1992; 71: 955-961.

38 Imai K, Dalal SS, Chen ES, et al. Human collagenase (matrix metalloproteinase-1) expression in the lungs of patients with emphysema. Am J Respir Crit Care Med 2001; 163: 786-791.

39 Segura-Valdez L, Pardo A, Gaxiola M, et al. Upregulation of gelatinases A and B, collagenases 1 and 2, and increased parenchymal cell death in COPD. Chest 2000; 117: 684-694.

40 Gosselink JV, Hayashi S, Elliott WM, et al. Differential expression of tissue repair genes in the pathogenesis of chronic obstructive pulmonary disease. Am J Respir Crit Care Med 2010; 181: 1329-1335.

$41 \mathrm{Xu} \mathrm{B}$, Chen $\mathrm{H}, \mathrm{Xu} \mathrm{W}$, et al. Molecular mechanisms of MMP9 overexpression and its role in emphysema pathogenesis of Smad3-deficient mice. Am J Physiol Lung Cell Mol Physiol 2012; 303: L89-L96.

42 Foronjy R, Nkyimbeng T, Wallace A, et al. Transgenic expression of matrix metalloproteinase- 9 causes adult-onset emphysema in mice associated with the loss of alveolar elastin. Am J Physiol Lung Cell Mol Physiol 2008; 294: L1149-L1157.

43 Churg A, Wang RD, Tai H, et al. Tumor necrosis factor- $\alpha$ drives $70 \%$ of cigarette smoke-induced emphysema in the mouse. Am J Respir Crit Care Med 2004; 170: 492-498.

44 Honey K, Benlagha K, Beers C, et al. Thymocyte expression of cathepsin L is essential for NKT cell development. Nat Immunol 2002; 3: 1069-1074.

45 Vivier E, Tomasello E, Baratin M, et al. Functions of natural killer cells. Nat Immunol 2008; 9: 503-510.

46 Prieto A, Reyes E, Bernstein ED, et al. Defective natural killer and phagocytic activities in chronic obstructive pulmonary disease are restored by glycophosphopeptical (inmunoferón). Am J Respir Crit Care Med 2001; 163: $1578-1583$

47 Nakagawa T, Roth W, Wong P, et al. Cathepsin L: critical role in Ii degradation and CD4 T cell selection in the thymus. Science 1998; 280: 450-453.

48 Zhang T, Maekawa Y, Sakai T, et al. Treatment with cathepsin L inhibitor potentiates Th2-type immune response in Leishmania major-infected BALB/c mice. Int Immunol 2001; 13: 975-982.

49 Sevenich L, Hagemann S, Stoeckle C, et al. Expression of human cathepsin L or human cathepsin V in mouse thymus mediates positive selection of T helper cells in cathepsin L knock-out mice. Biochimie 2010; 92: 1674-1680. Schurigt U, Eilenstein R, Gajda M, et al. Decreased arthritis severity in cathepsin L-deficient mice is attributed to an impaired T helper cell compartment. Inflamm Res 2012; 61: 1021-1029.

51 Funkelstein L, Beinfeld M, Minokadeh A, et al. Unique biological function of cathepsin L in secretory vesicles for biosynthesis of neuropeptides. Neuropeptides 2010; 44: 457-466.

52 Vatrella A, Montagnani S, Calabrese C, et al. Neuropeptide expression in the airways of COPD patients and smokers with normal lung function. J Biol Regul Homeost Agents 2010; 24: 425-432.

53 Iranmanesh A, Rochester DF, Liu J, et al. Impaired adrenergic- and corticotropic-axis outflow during exercise in chronic obstructive pulmonary disease. Metabolism 2011; 60: 1521-1529.

54 Cohly H, Stephens J, Markhov A, et al. Cell culture conditions affect LPS inducibility of the inflammatory mediators in J774A.1 murine macrophages. Immunol Invest 2001; 30: 1-15.

55 Tian J, Ishibashi K, Honda S, et al. The expression of native and cultured human retinal pigment epithelial cells grown in different culture conditions. Br J Ophthalmol 2005; 89: 1510-1517.

56 Neumann E, Riepl B, Knedla A, et al. Cell culture and passaging alters gene expression pattern and proliferation rate in rheumatoid arthritis synovial fibroblasts. Arthritis Res Ther 2010; 12: R83.

57 Reynier F, de Vos AF, Hoogerwerf JJ, et al. Gene expression profiles in alveolar macrophages induced by lipopolysaccharide in humans. Mol Med 2012; 18: 1303-1311.

58 Hu C, Wang J, Xu Y, et al. Current evidence on the relationship between five polymorphisms in the matrix metalloproteinases (MMP) gene and lung cancer risk: a meta-analysis. Gene 2013; 517: 65-71. 